Изв. АН Эстонии, Физ. Матем., 1989, 38, № 4, 368-371

удК 551.511 .3

P. РЫЫМ

\title{
ОБЩИЕ УРАВНЕНИЯ ДИНАМИКИ АТМОСФЕРЫ В ИЗОБАРИЧЕСКИХ КООРДИНАТАХ
}

\author{
(Представил Я. Эйнасто)
}

Уравнения движения идеальной атмосферы представлены в изобарической системе отсчета, при этом предложение о квазистатнчности не использовано.

Предложенная в $\left[{ }^{1}\right]$ идея представления уравнений динамики атмосферы в изобарических координатах оказалась очень плодотворной. В настоящее время изобарическая система координат, как и тесно связанная с ней сигма-система $\left[{ }^{2}\right]$, широко используется при численном моделировании динамики атмосферы.

До сих пор при преобразовании уравнений динамики в изобарическую систему всегда предполагалось, что атмосфера находится в квазистатическом равновесии и одно из уравнений движения можно заменить уравнением статики

$$
\frac{\partial p}{\partial z}=-g \varrho
$$

где $p$ - давление, $\varrho-$ плотность, $g$ - ускорение свободного падения, $z$ - высота в атмосфере над уровнем моря. Однако условие (1) не является неизбежной предпосылкой. Преобразование можно осуществить при гораздо менее ограничивающем условии монотонности изменения давления с высотой

$$
\frac{\partial p}{\partial z}<0
$$

Естественно, существуют процессы, при которых условие (2) будет нарушаться, например, распространение ударных волн, течения со субзвуковыми скоростями и т. п. Такие процессы в изобарической системе адекватно описать не удается. Однако в большинстве задач, представляющих интерес для физики атмосферы, условие (2) выполняется всегда и всюду.

В настоящей работе мы приводим уравнения динамики идеальной атмосферы (воздух считается свободным от внутреннего трения термодинамически идеальным газом) в изобарической системе при выполнении условия (2), т. е. без предположения о квазистатическом равновесии (1). Вывод уравнений мы опускаем и приводим только конечный результат.

Для краткости рассмотрим движение атмосферы над бесконечной равниной с подстилающей поверхностью на уровне моря. Независимыми переменными являются время $t$, горизонтальные координаты $x, y$ и давление $p$, причем

$$
0 \leqslant p \leqslant p_{0}(x, y, t),
$$

где $p_{0}(\cdot)-$ поле давления на поверхности, 
Динамическими полями, которые определяют состояние атмосферы, являются:

- высота изобарической поверхности $z(x, y, p, t)$,

- компоненты горизонтальной скорости

$$
u_{x}(x, y, p, t) \equiv \mathrm{d} x / \mathrm{d} t ; \quad u_{y}(x, y, p, t) \equiv \mathrm{d} y / \mathrm{d} t,
$$

- «вертикальная» скорость воздуха относительно криволинейной изобарической системы отсчета

$$
u_{p}(x, y, p, t) \equiv \mathrm{d} p / \mathrm{d} t,
$$

- поле температуры $T(x, y, p, t)$,

- поле давления на подстилающей поверхности $p_{0}(x, y, t)$,

- плотность в изобарическом пространстве $\varrho p(x, y, p, t)$, которая определяется инвариантно через элементарную массу $\mathrm{d} m$ при пөмощи равенства

$$
\mathrm{d} m=\varrho p \mathrm{~d} x \mathrm{~d} y \mathrm{~d} p
$$

и которая связана с обычной плотностью посредством соотношения

$$
\varrho p=-\varrho \frac{\partial z}{\partial p} \text {. }
$$

Уравнения движения для этих величин имеют вид

$$
\begin{gathered}
\varrho_{p} \frac{\mathrm{d}^{2} z}{\mathrm{~d} t^{2}}=1-g \varrho_{p}, \\
\varrho_{p} \frac{\mathrm{d} u_{x}}{\mathrm{~d} t}=-\frac{\partial z}{\partial x}+\varrho_{p} l u_{y} \\
\varrho_{p} \frac{\mathrm{d} u_{y}}{\mathrm{~d} t}=-\frac{\partial z}{\partial y}-\varrho_{p} l u_{x} \\
\frac{\mathrm{c}_{p} \frac{\mathrm{d} T}{\mathrm{~d} t}-R \frac{T u_{p}}{p}=w}{\mathrm{~d} t}+\varrho_{p}\left(\frac{\partial u_{x}}{\partial x}+\frac{\partial u_{y}}{\partial y}+\frac{\partial u_{p}}{\partial p}\right)=0 \\
\frac{\mathrm{d} p_{0}}{\mathrm{~d} t}-u_{p} \mid \frac{p=p_{0}}{\partial}=0 \\
\frac{\partial z}{\partial p}=-R \frac{\varrho_{p} T}{p} .
\end{gathered}
$$

Здесь $g$ - ускорение свободного падения, $l$ - параметр Кориолиса $(l=2 \omega \sin \varphi$, где $\omega-$ угловая скорость вращения Земли, $\varphi-$ географическая широта), $R$ и $c_{p}$ - газовые постоянные воздуха, w - плотность тепловых источников. Полная производная по времени определена следующим образом:

$$
\frac{\mathrm{d}}{\mathrm{d} t} \equiv \frac{\partial}{\partial t}+u_{x} \frac{\partial}{\partial x}+u_{y} \frac{\partial}{\partial y}+u_{p} \frac{\partial}{\partial p}
$$

Соотношение (10) является уравнением состояния идеального газа в изобарической системе отсчета. Это уравнение можно с учетом краевого условия $\left.z\right|_{p=p_{0}}=0$ интегрировать:

$$
z(x, y, p, t)=R \int_{p}^{p_{0}(x, y, t)} \frac{\varrho_{p} T}{p^{\prime}} \mathrm{d} p^{\prime} .
$$


Отметим, что вертикальная скорость $u_{p}$ входит в систему (4)-(10) неявно, для ее определения необходимо в каждый момент воспользоваться диагностическим уравнением (10) или его интегральным эквивалентом (11).

Уравнения квазистатики получим, если положим в уравнении (4) $\mathrm{d}^{2} z / \mathrm{d} t^{2}=0$ (сохраняя, однако, зависимость $z$ от времени $\left.t !\right)$. При этом (4) переходит в условие постоянства плотности при квазистатическом движении

$$
\mathrm{Q}_{p}=1 / \mathrm{g},
$$

а уравнение неразрывности (8) заменяется на условие бездивергентности трехмерного поля скорости

$$
\frac{\partial u_{x}}{\partial x}+\frac{\partial u_{y}}{\partial y}+\frac{\partial u_{p}}{\partial p}=0
$$

Остальные уравнения сохраняют свой прежний вид.

Динамическое уравнение (9) соответствует условию отсутствия вертикального движения в обыкновенном пространстве на уровне моря (в декартовой системе отсчета (9) имеет вид $\left.(\mathrm{d} z / \mathrm{d} t)_{z=0}=0\right)$. Это уравнение имеет простую физическую интерпретацию. В изобарическом пространстве атмосфера имеет свободную поверхность, уравнение для которой записывается в виде

$$
p=p_{0}(x, y, t) \text {. }
$$

Уравнение (9) описывает эволюцию этой поверхности.

Замена эволюционного уравнения (9) на диагностическое уравнение

$$
\left.u_{p}\right|_{p=p_{0}}=0
$$

с одновременной линеаризацией выражения (11) относительно отклонения $p_{0}(x, y, t)-\bar{p}(\bar{p}-$ стандартное давление на уровне моря) приводит к т. н. приближению твердого дна $\left[{ }^{3}\right]$.

Общие уравнения (4)-(11) способны, в отличие от уравнений квазистатики, описывать эффекты, связанные с отклонениями атмосферы от статического равновесия. Так, например, с помощью этих уравнений можно описать распространение акустических волн в атмосфере. В основном же система (4)-(11) имеет методологическое значение, так как позволяет более глубоко понять структуру изобарического координатного пространства и инвариантные свойства уравнений движения.

\section{ЛИТ ЕРАТУ РА}

1. Eliassen, A. // Geof. Publ., 1949, 17, № 3.

2. Philips, N. A. // J. Met., 1957, 14, № 2, 184-185.

3. Rõóm, R. // Preprint Acad. Sci. ESSR. Tallinn, 1988.

Институт астрофизики и физики атмосферы Академии наук Эстонской ССР

Поступила в редакцию $4 / \mathrm{I} \quad 1989$ 
R. $R O O M$

\section{OLDISED ATMOSFÄARI DUNAAMIKA VORRANDID ISOBAARSETES KOORDINAATIDES}

Ideaalse atmosfääri liikumisvõrrandid on teisendatud isobaarsetesse koordinaatidesse ilma kvaasistaatika eeldust kasutamata. Saadud võrrandid (4)-(11) kirjeldavad erinevalt kvaasistaatika vőrrandeist ka efekte, mis on seotud akustiliste häirituste levikuga atmosfääris.

\section{R. ROOM}

THE GENERAL FORM OF DYNAMICAL EQUATIONS OF THE ATMOSPHERE IN THE ISOBARIC COORDINATE SPACE

Equations of motion of the ideal atmosphere have been transferred from the Cartesian coordinates to the isobaric coordinate system without making use of the quasistatical equilibrium assumption. The obtained equations (4)-(11) are able to describe the effects connected with the propagation of the acoustical waves in the atmosphere. In the case of infinitely slow vertical motion, they obtain a common shape of primitive quasi-statical equations in the isobaric coordinate space. 